\title{
P038: Efficacy of prevention measures against nosocomial influenza at a large university hospital
}

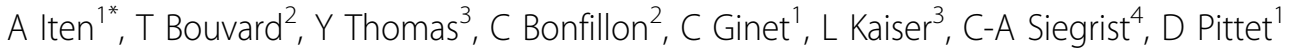 \\ From 2nd International Conference on Prevention and Infection Control (ICPIC 2013) \\ Geneva, Switzerland. 25-28 June 2013
}

\section{Introduction}

At HUG, healthcare workers (HCW) are currently obliged to be vaccinated or wear masks in ward corridors and patient rooms during seasonal influenza (SI) epidemics. Since winter 2011-12, HCWs vaccinated against SI wear a badge with the text "I am vaccinated to protect you" and, since winter 2012-13, those who are not vaccinated wear a badge with the text "I wear a mask to protect you". Regular audits have allowed to quantify compliance with recommendations in parallel with active recording of influenza cases.

\section{Methods}

During the SI epidemic, an audit recorded HCWs with a badge and those with correct mask wear over 2 periods of 2 weeks each in Feb-March 2012 and 3 periods of 2 weeks each in Jan-March 2013. Compliance was assessed as follows: (number of HCWs wearing a colored badge + number of HCWs wearing a mask correctly)/number of HCWs observed = number of compliant HCWs/number of HCWs observed, expressed as a percentage. Suspected cases of SI were confirmed by positive realtime RT-PCR reaction. Cases were defined as nosocomial (NOSO) SI when symptoms occurred $>72 \mathrm{~h}$ post-admission.

\section{Results}

Of 1390 HCWs observed in winter 2012, 469 wore a badge or mask (estimated compliance, 33.5\%). In winter 2013, 2070/2937 observed HCWs were compliant (70.5\%). We recorded 84 NOSO SI /152 SI (55.2\%) and 96 NOSO SI /267 SI (35.9\%) cases during 2011-12 and 2012-13, respectively. Compliance with recommendations in internal medicine averaged $68.6 \%$ in 2012 and
$72.2 \%$ in 2013. The proportion of NOSO SI cases remained stable (30.3\% and $21.6 \%$, respectively). At the geriatric hospital, compliance progressed from $58.6 \%$ to $72.2 \%$, while the proportion of NOSO SI cases decreased from $84.9 \%$ to $63.3 \%$, respectively. These measures prevented an estimated number of 115 NOSO SI cases at HUG in 2012-13, together with a reduced number of deaths among SI patients.

\section{Conclusion}

Mandatory badge wear, continuous SI epidemic surveillance and availability of compliance rates with recommendations decrease the risk of NOSO SI and improve patient safety.

\section{Disclosure of interest}

None declared.

\begin{abstract}
Author details
'Infection Control Program, University of Geneva Hospitals (HUG), Geneva, Switzerland. ${ }^{2}$ Department of Health Employees, University of Geneva Hospitals (HUG), Geneva, Switzerland. ${ }^{3}$ Laboratory of Virology, University of Geneva Hospitals (HUG), Geneva, Switzerland. ${ }^{4}$ Department of Child and Adolescent Medicine, University of Geneva Hospitals (HUG), Geneva, Switzerland.
\end{abstract}

Published: 20 June 2013

doi:10.1186/2047-2994-2-S1-P38

Cite this article as: Iten et al: P038: Efficacy of prevention measures against nosocomial influenza at a large university hospital. Antimicrobial Resistance and Infection Control 2013 2(Suppl 1):P38.

'Infection Control Program, University of Geneva Hospitals (HUG), Geneva,

Switzerland

Full list of author information is available at the end of the article

(c) 2013 Iten et al; licensee BioMed Central Ltd. This is an Open Access article distributed under the terms of the Creative Commons Attribution License (http://creativecommons.org/licenses/by/2.0), which permits unrestricted use, distribution, and reproduction in any medium, provided the original work is properly cited. 